\title{
Optimal control of a class of positive Markovian bilinear systems
}

\author{
Franco Blanchini ${ }^{1}$, Patrizio Colaneri ${ }^{2}$ and Richard H. Middleton ${ }^{3}$
}

\begin{abstract}
In this paper a class of input-parametrized bilinear positive Markovian systems is considered. The class considered has switching control of the diagonal entries of the dynamical matrix, together with Markov jump dynamics. This class is relevant to a variety of dynamical models arising in system biology and compartmental systems. It is proven that the any component of the expected value of the state vector is a convex functional of the input variables. If a linear cost of the final state is considered this implies that any Pontryagin solution of the associated optimal control problem is optimal and can be numerically computed by using standard gradient-type algorithms. Moreover, suboptimal strategies, both in open loop and closed loop are provided, mainly based on linear programming tools. An example is provided to illustrate the theory.
\end{abstract}

\section{INTRODUCTION}

This note deals with optimal control for a class of input-parametrized linear positive systems. The main motivation for this class lies in the observation that in many biological models the input represents a scheduling function that acts on the system parameters and has to be designed to minimize a cost representing the infection over a certain interval of time. The study of optimal control problems for such systems may then help inform application areas such as infection treatment protocols. For instance, in the recent work [16], a certain class of simplified HIV treatment switched models are considered in order to prolong the viral rebound and mitigate the infection effects. This class of problems also arise in certain

\footnotetext{
${ }^{1}$ Dipartimento di Matematica e Informatica, Università di Udine, 33100 Udine, Italy, email: blanchini@uniud.it

2 Dipartimento di Elettronica, Informazione e Bioingegneria, Politecnico di Milano, and IEIIT-CNR, 20133 Milano, Italy, email: patrizio.colaneri@polimi.it

${ }^{3}$ School of Electrical Engineering and Computer Science, the University of Newcastle, Callaghan, NSW 2308 Australia, email: richard.middleton@newcastle.edu.au; Research supported by an Australian Research Council Discovery Projects (DP130103039)
} 
types of epidemiological models, for example in SI (susceptible/infection) models (e.g. [20], [19]), at small initial infection stage.

The class of systems considered is related to that of switched/hybrid systems, for which the optimal control problem has been widely studied [8], [12] and a variational approach has been developed by [21] and [18]. The optimal control problem is inherently nonlinear and therefore non-convex in general. There is therefore no guarantee that solutions satisfying the Pontryagin maximum principle provide globally optimal solutions. Moreover, finding such solutions is in general a formidable task, since it requires backward and forward iterations of the state-costate dynamics with no guarantee of convergence. Convexity of the cost with respect to the control function is therefore a strong property that ensures optimality of the Pontryagin solutions of the average deterministic system and allows the use of simple interior point algorithms to find an optimal solution.

In the deterministic setting, this problem has been tackled in [26], [2] where it was shown that for a class of input parametrized bilinear positive systems a convex functional of the final state is indeed convex with respect to the control variables. In this note we prove that this property also holds for the expected value of the cost when this class is extended to include Markovian jump switchings in addition to controlled switching of diagonal elements of the dynamical matrix, see [3] for a recent monograph on positive Markov jump linear systems. For the case of infinite time horizon, when time-driven optimal control laws may prove impractical, suboptimal control strategies are provided in state-feedback form. This form also sheds some light on stability and stabilizability problems for deterministic switched positive systems, see [4] for a recent monograph on switched linear positive systems.

The paper is organized as follows. The class of systems and the associated optimal control problem are defined in Section 2. In this section, the result on convexity is introduced and also some hints on the possible algorithms are given together with possible important extensions on the class of considered cost functions that preserve convexity. In Section 3 time-driven and state-feedback suboptimal strategies are provided, both in the deterministic and stochastic settings. Section 4 includes an example based on the epidemiological model in [9], extended so as to include Markovian switching.

Before proceeding we introduce some basic notation. $\mathbb{R}_{+}$is the semiring of nonnegative real numbers. A matrix or vector, $A$, with entries in $\mathbb{R}_{+}$is a nonnegative matrix (or vector). 
The matrix $A$ is positive $(A>0)$ if it is nonnegative and nonzero, and strictly positive $(A \gg 0)$ if all its entries are positive. A square matrix $A=\left[a_{i j}\right]$ is said to be Metzler if its off-diagonal entries are nonnegative, namely $a_{i j} \geq 0$ for every $i \neq j$. An $n \times n$ Metzler matrix $A$, with $n>1$, is reducible if there exists a permutation matrix $P$ such that

$$
P^{\prime} A P=\left[\begin{array}{cc}
A_{11} & A_{12} \\
0 & A_{22}
\end{array}\right],
$$

where $A_{11}$ is a $k \times k$ matrix, $1 \leq k \leq n-1$. A Metzler matrix which is not reducible is called irreducible. For an irreducible Metzler matrix $A$ it is know that $\max \{\operatorname{Re}(\lambda): \lambda \in \sigma(A)\}$ is a simple eigenvalue of $A$, called the Perron-Frobenius eigenvalue and denoted by $\lambda_{f}$, and that the corresponding eigenspace is generated by a strictly positive eigenvector, called the Perron-Frobenius eigenvector. For further details on positive systems the reader is referred to [14]. $\mathbf{1}_{n}$ is the $n$-dimensional vector with all entries equal to 1 . The suffix $n$ will be omitted when the vector size is clear from the context. The convex polytope of the nonnegative $m$-tuples, $M \in \mathbb{N}$, that sum to 1 will be denoted by:

$$
\mathcal{U} \doteq\left\{\alpha \in \mathbb{R}_{+}^{m}: \sum_{i=1}^{m} \alpha_{i}=1\right\}=\left\{\alpha \in \mathbb{R}_{+}^{m}: \mathbf{1}^{\prime} \alpha=1\right\} .
$$

The symbol $\mathcal{G}_{m}$ indicates the set of $m \times m$ Meztler matrices $A$ such that $A \mathbf{1}_{m}=0$. Therefore matrices in $\mathcal{G}_{m}$ can be interpreted as rate transition probability matrices of a Markov chain, see [11].

A function $V: \mathbb{R}^{n} \rightarrow \mathbb{R}$ is said to be co-positive if $V(x)>0$ for every $x>0$ and $V(0)=0$. A linear co-positive function takes the form $V(x)=c^{\prime} x$, for some $c \in \mathbb{R}_{+}^{n}, c \gg 0$. For Markovian switching systems see e.g. [13].

\section{Optimal COntrol PRoblem}

Consider a nonlinear system described by

$$
\dot{x}=\left(M_{\sigma}+\Lambda_{\sigma}(u)\right) x, \quad x(0)=x_{0}
$$

where $x(t)$ is the $n$-dimensional state vector, $\sigma(t)$, taking values in $\mathcal{N}=\{1,2, \ldots, N\}$, is a stochastic process governed by a Markov chain with rate transition probability matrix, say $\Pi \in \mathcal{G}_{N}$, the matrices $M_{i}, i=1,2, \cdots, N$ are Metzler, $\Lambda_{i}(u), i=1,2, \cdots, m$ are 
$n \times n$ diagonal matrices of convex and continuous functions of $u$ and $u(t)$, for each $t$, is a $m$-dimensional control variable belonging to the polytope $\mathcal{U} \doteq\left\{u \in \mathbb{R}_{+}^{m}: \mathbf{1}^{\prime} u=1\right\}$. We assume further that $u$, as a function of time, belongs to the functional space $\mathcal{U}_{s}$ of piecewise continuous functions taking values in $\mathcal{U}$, in the interval $\left[0, t_{f}\right]$ where $t_{f}>0$ is a given final time instant. Finally, for simplicity, the initial state $x(0)=x_{0}$ is assumed to be a deterministic nonnegative vector, i.e. $x_{0} \in \mathbb{R}_{+}^{n}$.

Since $A_{i}(u)=M_{i}+\Lambda_{i}(u)$ is Metzler for any $u \in \mathcal{U}$ and $i \in \mathcal{N}, \mathbb{R}_{+}^{n}$ is positively invariant for any $u \in \mathcal{U}_{s}$ and $\sigma(t)$, i.e. the system is positive.

Remark 1: The class of systems described by (1) includes Markov jump processes in a polytope, described by

$$
\dot{x}=\left(M_{\sigma}+\sum_{j=1}^{m} \Lambda_{\sigma}^{[j]} u_{j}\right) x
$$

with $u \in \mathcal{U}_{s}$ and $\Lambda_{i}^{[j]}, i \in \mathcal{N}, j \in \mathcal{M}$ are diagonal matrices. This system can be considered to be the embedding of a positive Markov Jump linear switched system. In this case $\Lambda_{i}(u)=\Lambda_{i}^{[\gamma]}$ where the switching signal $\gamma$ is a left continuous piecewise constant signal taking values in the finite set $\mathcal{M}=\{1,2, \cdots, m\}$ so that $u_{i}(t)=1$ if $\gamma(t)=i$ and $u_{i}(t)=0$ otherwise.

For instance, such a system can represent a simplified model of treatment of HIV infection dynamics where the mutation rate is uncertain and modeled according to a Markovian jump parameter (see for example [16] for a fixed mutation rate), where $x$ represents the concentrations of various viral mutants in a patient, and $\gamma$ represents the selection of a suitable therapy. Other examples can be found in the widespread SIR (or SI, or SIRS) models of epidemiology over a network of uncertain infection rates, in the initial infection phase (see for example [20], [19] for fixed infection rates). The embedded system is important in the context of optimal control, since it is capable of capturing possible singular arcs corresponding to sliding trajectories of the expected value of the system state.

We now considered an optimal control problem for the system (1), with terminal cost functional:

$$
J\left(u, x_{0}\right):=E\left[c_{\sigma\left(t_{f}\right)}^{\prime} x\left(t_{f}\right)\right]
$$

where $c_{i}, i \in \mathcal{N}$ are nonnegative vectors and the expectation is performed with respect to the stationary probability measure of the stochastic process $\sigma$. 
From now on we assume that transition rate matrix $\Pi \in \mathcal{G}_{N}$ is irreducible, so that the stationary probability measure $\bar{\pi} \in \mathcal{U}$ is the unique equilibrium point in $\mathcal{U}$ of the Kolmogorov equation $\dot{\pi}=\Pi^{\prime} \pi$ (Frobenius eigenvector), satisfying $\Pi^{\prime} \bar{\pi}=0$. Moreover, the entries of $\bar{\pi}$ are all strictly positive. Performing the expectation with respect to $\bar{\pi}$ means to initialize the system with $\pi(0)=\bar{\pi}$ so that $\pi(t)=\bar{\pi}, \forall t \geq 0$.

Finally, as said above, the initial state $x_{0}$ is assumed to be a deterministic fixed (yet arbitrary) vector. The problem is to find $u^{o} \in \mathcal{U}_{s}$ that minimizes the cost, i.e. $\min _{u \in \mathcal{U}_{s}} J\left(u, x_{0}\right)$. We can first write equations for the propagation of the expectation of the state variable. To this end, define the vectors

$$
s_{i}(t)=E[x(t) \mid \sigma(t)=i] \bar{\pi}_{i}, \quad i \in \mathcal{N}
$$

where $\bar{\pi}_{i}, i=1,2, \cdots, N$ are the elements of the stationary measure $\bar{\pi} \in \mathcal{U}$ associated with the Markov chain. It is a simple computation (see [1] for details) to show that the following propagation equation for the mean of the state variable holds:

$$
\dot{s}_{i}(t)=\left(M_{i}+\Lambda_{i}(u)\right) s_{i}(t)+\sum_{j=1}^{N} \pi_{j i} s_{j}(t), \quad s_{i}(0)=\bar{\pi}_{i} x_{0}
$$

where $\pi_{i j}, i \in \mathcal{N}, j \in \mathcal{N}$, are the entries of $\Pi$. Moreover, $E[x(t)]=\sum_{i=1}^{N} s_{i}(t)$. As for (3), thanks to the law of total expectation, it follows

$$
E\left[c_{\sigma\left(t_{f}\right)}^{\prime} x\left(t_{f}\right)\right]=\sum_{i=1}^{N} E\left[c_{i}^{\prime} x\left(t_{f}\right) \mid \sigma\left(t_{f}\right)=i\right] \bar{\pi}_{i}=\sum_{i=1}^{N} c_{i}^{\prime} E\left[x\left(t_{f}\right) \mid \sigma\left(t_{f}\right)=i\right] \bar{\pi}_{i}
$$

Therefore, our stochastic optimal control problem becomes that of minimizing

$$
J\left(u, x_{0}\right):=\bar{c}^{\prime} s\left(t_{f}\right)
$$

under the action of the deterministic $n N$ dimensional dynamical system (4), compactly rewritten as

$$
\dot{s}=(\bar{M}+\bar{\Lambda}(u)) s, \quad s(0)=\bar{s}_{0}
$$


where

$$
\begin{aligned}
\bar{s}^{\prime} & =\left[\begin{array}{llll}
s_{1}^{\prime} & s_{2}^{\prime} & \cdots & s_{N}^{\prime}
\end{array}\right] \\
\bar{c}^{\prime} & =\left[\begin{array}{llll}
c_{1}^{\prime} & c_{2}^{\prime} & \cdots & c_{N}^{\prime}
\end{array}\right] \\
\bar{M} & =\left[\begin{array}{cccc}
M_{1} & 0 & \cdots & 0 \\
0 & M_{2} & \cdots & 0 \\
\vdots & \vdots & \ddots & \vdots \\
0 & 0 & \cdots & M_{N}
\end{array}\right]+\Pi^{\prime} \otimes I_{n} \\
\bar{\Lambda}(u)= & {\left[\begin{array}{cccc}
\Lambda_{1}(u) & 0 & \cdots & 0 \\
0 & \Lambda_{2}(u) & \cdots & 0 \\
\vdots & \vdots & \ddots & \vdots \\
0 & 0 & \cdots & \Lambda_{N}(u)
\end{array}\right] } \\
\bar{s}_{0}= & \bar{\pi} \otimes x_{0}
\end{aligned}
$$

A first result concerns the characterization of the cost in terms of the extended system (6) and its adjoint.

Theorem 1: Consider system (1). For any $u \in \mathcal{U}_{s}$ it follows that

$$
E\left[c_{\sigma\left(t_{f}\right)}^{\prime} x\left(t_{f}\right)\right]=\sum_{i=1}^{N} \bar{\pi}_{i} r_{i}(0)^{\prime} x_{0}=\sum_{i=1}^{N} c_{i}^{\prime} s_{i}\left(t_{f}\right)
$$

where

$$
\begin{array}{ll}
0=\dot{r}_{i}(t)^{\prime}+r_{i}(t)^{\prime} A_{i}(u(t))+\sum_{j=1}^{N} \pi_{i j} r_{j}(t)^{\prime}, & r_{i}\left(t_{f}\right)=c_{i} \\
0=-\dot{s}_{i}(t)+A_{i}(u(t)) s_{i}(t)+\sum_{j=1}^{N} \pi_{j i} s_{j}(t), & s_{i}(0)=x_{0} \bar{\pi}_{i}
\end{array}
$$

Proof: The equality between the two summations in (7) follows by noticing that

$$
\sum_{i} d / d t\left(r_{i}^{\prime} s_{i}\right)=0
$$

Using the law of total expectation the proof is concluded.

Note that the function $V(x, \sigma, t)=r_{\sigma}(t)^{\prime} x$ acts as a stochastic Lyapunov function for the 
cost computation. Indeed, compute the infinitesimal generator at time $t$ with the position $\sigma(t)=s, x(t)=x$. Denote by $\mathcal{E}_{s}$ the event $\sigma(t)=s$ and by $\mathcal{E}_{x s}$ the event $(x(t), \sigma(t))=(x, s)$. It follows that

$$
\begin{aligned}
\mathcal{L} V(x, s) & =\lim _{h \rightarrow 0}\left(\frac{1}{h} E\left[V(x(t+h), \sigma(t+h), t+h) \mid \mathcal{E}_{x s}\right]-V(x, s, t)\right) \\
& =\lim _{h \rightarrow 0}\left(\frac{1}{h} E\left[r_{\sigma(t+h)}(t+h)^{\prime} x(t+h) \mid \mathcal{E}_{x s}\right]-\left(r_{s}(t)^{\prime} x\right)\right) \\
& =\lim _{h \rightarrow 0}\left(\frac{1}{h} E\left[r_{\sigma(t+h)}(t+h)^{\prime}\left(I+A_{s}(u) h\right) x \mid \mathcal{E}_{s}\right]-\left(r_{s}(t)^{\prime} x\right)\right)
\end{aligned}
$$

Now notice that

$$
E\left[r_{\sigma(t+h)}(t+h) \mid \mathcal{E}_{s}\right]=h \sum_{i \neq s} \pi_{s i} r_{i}(t+h)+\left(1-\pi_{s s}\right) r_{s}(t+h)
$$

Therefore

$$
\mathcal{L} V(x, s)=\left(\dot{r}_{s}\right)^{\prime} x+\left(r_{s}\right)^{\prime} A_{s}(u) x+\sum_{i=1}^{N} \pi_{s i}\left(r_{i}\right)^{\prime} x=0
$$

yields $E\left[c_{\sigma\left(t_{f}\right)}^{\prime} x\left(t_{f}\right)\right]=E\left[r_{\sigma(0)}(0)^{\prime} x_{0}\right]=\sum_{i=1}^{N} \bar{\pi}_{i} r_{i}(0)^{\prime} x_{0}$.

Notice that system (9) represents again the extended system (6), whereas (8) is its adjoint, that can be compactly written as

$$
\dot{r}=-\bar{A}(u)^{\prime} r, \quad r\left(t_{f}\right)=\bar{c}
$$

where $\bar{A}(u)=\bar{M}^{\prime}+\bar{\Lambda}(u)^{\prime}$. The adjoint system plays an important role for the statement of necessary condition for optimality. In the literature on optimal control, direct sufficient conditions (for instance associated with Hamilton-Jacobi-Bellman equations) are often impractical. On the other hand, numerical solutions can be computed looking at necessary solutions for optimality. We therefore define a Pontryagin solution as a candidate optimal control solution satisfying the necessary conditions. For further details see e.g. [7].

Definition 1: A triple $u^{o}(t), s^{o}(t), r^{o}(t)$, that satisfies (for almost all $t$ ) the system of 
equations:

$$
\begin{aligned}
\dot{s}^{o}(t) & =\bar{A}\left(u^{o}(t)\right) s^{o}(t) \\
-\dot{r}^{o}(t) & =\bar{A}\left(u^{o}(t)\right)^{\prime} r^{o}(t) \\
u^{o}(t) & \in \underset{u \in \mathcal{U}}{\operatorname{argmin}}\left\{r^{o \prime}(t) \bar{A}(u) s^{o}(t)\right\}
\end{aligned}
$$

with boundary conditions $s^{o}(0)=\bar{s}_{0}, r^{o}\left(t_{f}\right)=\bar{c}$, is called a Pontryagin solution for the optimal control problem (5), (6).

We are now ready to state an important result of the paper, namely the convexity of the $\operatorname{map} u \in \mathcal{U}_{s} \rightarrow J\left(u, x_{0}\right) \in \mathbb{R}_{+}$.

Theorem 2: Consider the system (1) and assume that $M_{i}, i \in \mathcal{N}$ are Metzler matrices, $\Lambda_{i}(u), i \in \mathcal{N}$ diagonal matrices composed of convex functions of $u \in \mathcal{U}$, and consider the cost function (3), where $c_{i}, i \in \mathcal{N}$ are nonnegative vectors. Then, the functional $u \rightarrow$ $E\left[c_{\sigma\left(t_{f}\right)}^{\prime} x\left(t_{f}\right)\right]$ from $\mathcal{U}_{s}$ to $\mathbb{R}_{+}$is convex.

Proof: Notice first that the cost cost given by (3) can be written as (5) where $s(t)$ obeys to (6). This is a deterministic system with $\bar{M}$ Metzler and $\bar{\Lambda}(u)$ diagonal matrix of continuous and convex functions of $u$. Therefore the proof is immediate from Theorem 1 in [2] since the optimal control problem for the stochastic systems (1) with cost given by (3) is equivalent to the optimal control problem for the deterministic system (6) with cost (5).

However, to stress the importance of the previous result, the following theorem stating the optimality of a Pontragyn solution associated with the optimal control problem is provided, see Theorem 2 in [2] for a proof.

Theorem 3: Consider (1) and assume that $M_{i}$ are Metzler, $\Lambda_{i}(u)$ are diagonal matrices composed of convex functions of $u \in \mathcal{U}$, and consider the cost function (3), where $c_{i}$ are nonnegative vectors. Then the optimal control problem admits at least one Pontryagin solution $\left(u^{o}, s^{o}, r^{o}\right)$ and $u^{o}$ is a global optimal control signal relative to $x_{0}$. Moreover any Pontryagin solution is a global optimum, and the value of the optimal cost functional is $r^{o \prime}(0) \bar{s}_{0}$.

Remark 2: The theorem above ensures that under the convexity assumption of the functions in $\Lambda_{i}(u)$, the cost functional is convex as well and hence the set of Pontryagin solutions is a convex set. Strict convexity of $\Lambda_{i}(u)$ does not directly imply strict convexity of the cost 
functional (and hence uniqueness of the optimal solution).

Remark 3: The expectated value of the stochastic process $x(t)$ is made with respect to the stationary probability measure. It is not difficult to consider instead the probability measure depending on time according to the (Kolmogorov) differential equation $\dot{\pi}=\Pi^{\prime} \pi$ and a given initial probability vector $\pi(0)$.

We end this section by providing a computational scheme for the optimal control problem. The convexity property allows using different types of algorithms to find the solution of

$$
\min _{u \in \mathcal{U}_{s}} J\left(u, x_{0}\right), \quad J\left(u, x_{0}\right)=\bar{c}^{\prime} s\left(t_{f}\right)
$$

Computations can be cast in discrete-time, by taking, as done in the previous section, a subdivision of the interval $\left[0, t_{f}\right]$ into $L$ intervals $T_{1}, T_{2}, \cdots, T_{L}$. The control variable may be approximated as piecewise constant, i.e. ( with $T_{0}=0, T_{L}=t_{f}$ ):

$$
u(t)=\bar{u}^{[k]}, \quad t \in\left[\sum_{i=1}^{k-1} T_{i}, \sum_{i=1}^{k-1} T_{i}+T_{k}\right)
$$

The discretized control is denoted by $\bar{u}$ taking values in $\overline{\mathcal{U}}$, the cartesian products of $\mathcal{U}$. Hence the problem is to find

$$
\min _{u \in \overline{\mathcal{U}}} J\left(\bar{u}, x_{0}\right)=\min _{u \in \overline{\mathcal{U}}} \bar{c}^{\prime} \prod_{i=L}^{1} e^{\left(\bar{M}+\bar{\Lambda}\left(\bar{u}^{[i]}\right)\right) T_{i}} x_{0}
$$

The constrained optimization problem can be solved using the standard Matlab function fmincon.m which is based on an interior point method. Notice indeed that $\overline{\mathcal{U}}$ is a convex set and that $J\left(\bar{u}, x_{0}\right)$ is a convex function of $\bar{u}$, recall Theorem 2 .

To further assist the convex minimisation, note that we can explicitly compute the gradient of the cost. The gradient of $J\left(\bar{u}, x_{0}\right)$ is a $m L$-dimensional row vector and the $j^{\text {th }}$ $m$-dimensional row entry $g^{[j]}(k)$ can be computed from (14). Indeed a simple computation shows that the generic $r$-th entry $g_{r}^{[j]}(k)$ of $g^{[j]}$ is

$$
g_{r}^{[j]}(k)=c^{\prime} \Psi_{r}^{[j]}\left(\left.\Phi_{r}^{[j]}\right|_{\bar{u}(k)}\right) \Gamma_{r}^{[j]} x_{0}
$$


where

$$
\Psi_{r}^{[j]}=\prod_{i=L}^{j+1} e^{\left(\bar{M}+\bar{\Lambda}\left(\bar{u}^{[i]}\right)\right) T_{i}}, \Gamma_{r}^{[j]}=\prod_{i=j-1}^{1} e^{\left(\bar{M}+\bar{\Lambda}\left(\bar{u}^{[i]}\right)\right) T_{i}}
$$

and

$$
\begin{aligned}
\Phi_{r}^{[j]} & =\frac{\partial e^{\left(\bar{M}+\bar{\Lambda}\left(\bar{u}^{[j]}\right)\right) T_{j}}}{\partial \bar{u}_{r}^{j}} \\
& =\int_{0}^{T_{j}} e^{\left(\bar{M}+\bar{\Lambda}\left(\bar{u}^{[j]}\right)\right)(t-\tau)} \frac{\partial \bar{\Lambda}\left(\bar{u}^{[j]}\right)}{\partial \bar{u}_{r}^{[j]}} e^{\left(\bar{M}+\bar{\Lambda}\left(\bar{u}^{[j]}\right)\right) \tau} d \tau \\
& =\left(\begin{array}{ll}
0 & I
\end{array}\right) e^{\left(\begin{array}{cc}
\bar{M}+\bar{\Lambda}\left(\bar{u}^{[j]}\right) & 0 \\
\frac{\partial \Lambda\left(\bar{u}^{[j]}\right)}{\partial \bar{u}_{r}^{[j]}} & \bar{M}+\bar{\Lambda}\left(\bar{u}^{[j]}\right)
\end{array}\right) T^{T_{j}}}\left(\begin{array}{l}
I \\
0
\end{array}\right)
\end{aligned}
$$

Remark 4: Notice that the gradient $g_{r}^{[j]}(k)$ can be equivalently computed via discretization by integrated backward the co-state equation (10) along with the differential equation for $\frac{\partial r}{\partial u}$.

Remark 5: Notice that the algorithm can be further enhanced by explicit computation of the Hessian matrix.

As a last observation, notice that the optimal cost $J\left(u^{o}, x_{0}\right)$ is a concave function of $\bar{s}_{0}$ and hence of $x_{0}$, see for instance the recent papers [15] and [5]. Then taking $x_{0} \in \mathcal{U}$ for some predefined set of initial states, it may also be of interest to find a saddle point solution of the min-max problem

$$
\min _{u \in \mathcal{U}_{s}} \max _{x_{0} \in \mathcal{U}} J\left(u, x_{0}\right)
$$

i.e. a solution $u^{*}, x_{0}^{*}$ such that $J\left(u^{*}, x_{0}\right) \leq J\left(u^{*}, x_{0}^{*},\right) \leq J\left(u, x_{0}^{*}\right)$ for any $x_{0} \in \mathcal{U}$ and any $u \in \mathcal{U}_{s}$. In this regard, taking again the above discretization of $u$, we are able to write the computational scheme

$$
\begin{aligned}
\bar{u}^{[k+1]} & =\operatorname{Proj}_{\overline{\mathcal{U}}}\left(\bar{u}^{[k]}-\alpha g^{[k]}\right) \\
\bar{x}_{0}^{[k+1]} & =\operatorname{Proj}_{\mathcal{A}}\left(\bar{x}_{0}^{[k]}+\alpha h^{[k]}\right)
\end{aligned}
$$

where $\operatorname{Proj}_{\mathcal{U}}$ is the projection on $\mathcal{A}, h^{[k]}$ is the gradient of $J\left(u, x_{0}\right)$ with respect to $x_{0}$ at the $k$-th iteration. The vector $h$ can be easily computed (thanks to the linearity of $J\left(u, x_{0}\right)$ with 
respect to $x_{0}$, as $h=\bar{c}^{\prime} \prod_{i=L}^{1} e^{\left(\bar{M}+\bar{\Lambda}\left(\bar{u}^{[i]}\right)\right) T_{i}}$.

\section{A. Extensions}

The proof of convexity has been carried out by looking at a problem where the cost is a generic linear combination of the final state. However we know that any convex and nondecreasing function of a convex function is convex, and hence a similar analysis can be carried out considering such a cost. Linearity of the cost with respect to $s\left(t_{f}\right)$ and linearity of the system for any given control function also implies linearity with respect to the initial state, so implying concavity of the optimal cost with respect to $x_{0}$. Notice that to preserve concavity it is possible to consider a cost that is a concave and nonincreasing function of the state.

Note also that the cost functional can be readily extended to an integral cost of the form

$$
J\left(u, x_{0}\right)=E\left[c_{\sigma\left(t_{f}\right)}^{\prime} x\left(t_{f}\right)+\int_{0}^{t_{f}} d_{\sigma(t)}^{\prime} x(t) d t\right]
$$

where $d_{i}, i \in \mathcal{N}$ are nonnegative vectors. Indeed the former problem (1) and cost (3) are

recovered by augmenting the state $\xi=\left[x^{\prime} \eta\right]$, with $\eta=\int_{0}^{t} d_{\sigma(\tau)}^{\prime} x(\tau) d \tau$ so that $J\left(u, x_{0}\right)=$ $E\left[\left[\begin{array}{cc}c_{\sigma}^{\prime} & 1\end{array}\right] \xi\left(t_{f}\right)\right]$.

Following a similar rationale we can also establish an extension of the presented theory for systems affected by a constant input, i.e. $\dot{x}=A_{\sigma}(u) x+b_{\sigma}$, where $b_{i}, i \in \mathcal{N}$ are nonnegative vectors. This can be achieved again by state augmentation, i.e. $\xi=\left[x^{\prime} z\right]$, with $\dot{z}=0$, $z(0)=1$ and $\dot{x}=A_{\sigma}(u) x+b_{\sigma} z$, so that $J\left(u, x_{0}\right)=\left[\begin{array}{ll}c^{\prime} & 0\end{array}\right] \xi\left(t_{f}\right)$.

Finally, notice that the convexity property also holds when the cost is enriched by an additional term that depends on the control variable $u \in \mathcal{U}_{s}$, say $h_{\sigma}(u)$, provided that $h_{i}(u)$, $i \in \mathcal{N}$, are convex functionals of $u$.

Therefore, our convexity properties holds when the cost is

$$
J\left(u, x_{0}\right)=E\left[c_{\sigma\left(t_{f}\right)}^{\prime} x\left(t_{f}\right)+\int_{0}^{t_{f}} d_{\sigma}^{\prime} x(t) d t+h_{\sigma(t)}(u)\right]
$$

under the assumption that, for each $i$, the system structure $\dot{x}=\left(M_{\sigma}+\Lambda_{\sigma}(u)\right)$ is such that $\Lambda_{i}(u)$ are diagonal matrices with convex function of $u, M_{i}$ are Metzler matrices and $h_{i}$ are convex functionals of $u$. 
Remark 6: It is important to note that, in the derivations above, the open loop control action is thought to be a deterministic time vector. It is clear that any feedback strategy based on the measurement of $x$ and/or $\sigma$ results in a stochastic (dependent on $\sigma$ ) control action.

\section{State FEedback CONTROL FOR POSitive DUAL SWITChed SYSTEMS}

In this section we consider the class of systems described by the equations

$$
\dot{x}=\left(M_{\sigma}+\Lambda_{\sigma}^{[\gamma]}\right) x
$$

where $M_{i}, i \in \mathcal{N}$ are Metzler matrices and $\Lambda_{i}^{[j]}, i \in \mathcal{N}, j \in \mathcal{M}$, are diagonal matrices. Again $\sigma(t)$ is a Markovian stochastic switching signal and $\gamma(t)$ is a control switching signal. Associated with (18), with a slight abuse of notation, we consider the cost function

$$
J\left(\gamma, x_{0}\right)=E\left[c_{\sigma\left(t_{f}\right)}^{\prime} x\left(t_{f}\right)\right]
$$

where $c_{i}>0, i \in \mathcal{N}$.

Remark 7: System (1) can be considered a relaxation of (18) where $\Lambda_{i}(u)=\sum_{j=1}^{m} \Lambda_{i}^{[j]} u_{j}$, see Remark 1, in the sense that $\gamma(t)=k$ in (18) corresponds $u(t)=e_{k}$ in (2). In the literature of switching systems, system (18) is referred to as a positive dual switching system, see [23] and Chapter 3 in [22].

In the previous chapter we have discussed the open-loop optimal control problem for the class of systems described by equation (1), that also includes the class of dual switching systems (18). In the following we provide suboptimal control strategies for (18), (19), based on the knowledge of $\sigma(t)$ (mode-dependent) and $x(t)$ (state-feedback). A remark is dedicated to the important subject of mode-independent control. Notice that the strategies discussed below do not exploit the convexity of the cost with respect to the elements of matrices $\Lambda_{i}^{[j]}$. This issue is worth of further consideration in future investigations.

\section{A. $\gamma(t)$ as a function of both $x(t)$ and $\sigma(t)$}

The following result provides a suboptimal strategy in feedback form, see [6] for a similar result for infinite horizon cost and non positive systems. In the theorem above, $A_{i}^{[j]}:=$ $M_{i}+\Lambda_{i}^{[j]}$ 
Theorem 4: Take any Metzler matrix $\Phi=\left[\phi_{i j}\right] \in \mathcal{G}_{m}$ and consider the solutions $q_{i}^{[j]}(t)$, $i \in \mathcal{N}, j \in \mathcal{M}$ satisfying

$$
0=\dot{q}_{i}^{[j]}(t)^{\prime}+q_{i}^{[j]}(t)^{\prime} A_{i}^{[j]}+\sum_{k=1}^{N} \pi_{i k} q_{k}^{[j]}(t)^{\prime}+\sum_{r=1}^{m} \phi_{j r} q_{i}^{[r]}(t), \quad q_{i}^{[j]}\left(t_{f}\right)=c_{i}, \quad \forall j
$$

The control law

$$
\gamma^{*}(t)=g(t, x, \sigma)=\arg \min _{r} q_{\sigma}^{[r]}(t)^{\prime} x(t)
$$

is such that

$$
J\left(\gamma^{*}, x_{0}\right)<\min _{r} \sum_{i=1}^{N} \bar{\pi}_{i} q_{i}^{[r]}(0)^{\prime} x_{0}
$$

Proof: Consider the stochastic Lyapunov function $V(x, s, t)=\min _{r} q_{s}{ }^{[r]}(t)^{\prime} x$ and compute the corresponding infinitesimal generator at time $t$ with the positions $x(t)=x, \sigma(t)=s$ and $g=\operatorname{argmin}_{r} q_{i}^{[j]}(t)^{\prime} x$. For brevity, the event $\sigma(t)=s$ and the joint event $(x(t), \sigma(t))=$ $(x, s)$ will be indicated by $\mathcal{E}_{s}$ and $\mathcal{E}_{x, s}$, respectively. It follows that:

$$
\begin{array}{r}
\mathcal{L} V(x, s, t)=\lim _{h \rightarrow 0} \frac{1}{h}\left(E\left[V\left(x(t+h), \sigma(t+h) \mid \mathcal{E}_{x, s}\right]-V(x, s)\right)\right. \\
=\lim _{h \rightarrow 0} \frac{1}{h}\left(E\left[\min _{r} q_{\sigma(t+h)}^{[j]}(t+h)^{\prime} x(t+h) \mid \mathcal{E}_{x, s}\right]-\min _{r} q_{s}^{[r]} x\right) \\
=\lim _{h \rightarrow 0} \frac{1}{h}\left(E\left[\min _{r} q_{\sigma(t+h)}^{[j]}(t+h)^{\prime}\left(I+A_{s}^{g} h\right) x \mid \mathcal{E}_{s}\right]-x^{\prime} P_{s}^{g} x\right)
\end{array}
$$

Notice now that the expected value of the minimum of a function is not greater than the minimum of the expectation. Moreover,

$$
E\left[q_{\sigma(t+h)}^{[r]}(t+h) \mid \mathcal{E}_{s}\right]=h \sum_{j \neq s} \pi_{s j} q_{j}^{[r]}(t+h)+\left(1+\pi_{s s} h\right) q_{s}^{[r]}(t+h)
$$

Therefore, by neglecting the terms of order $o(h)$, it follows that

$$
\mathcal{L} V(x, s, t) \leq \lim _{h \rightarrow 0} \frac{1}{h}\left(\min _{r} f_{r}(t+h)^{\prime} x-q_{s}^{[g]}(t) x\right)
$$

where

$$
f_{r}(t)=h\left(\sum_{j} \pi_{s j} q_{j}^{[r]}(t)+\left(A_{s}^{g}\right)^{\prime} q_{s}^{[r]}(t)\right)+q_{s}^{[r]}(t)
$$


Now, since the function $f_{r}(t+h) x$ evaluated at $r=g$ is not less than its minimum, i.e. $f_{g}(t+h) x \geq \min _{r} f_{r}(t+h) x$, we obtain

$$
\mathcal{L} V(x, s, t) \leq\left(\dot{q}_{s}^{[g]}(t)^{\prime}+q_{s}^{[g]}(t)^{\prime} A_{s}^{[g]}+\sum_{j} \pi_{s j} q_{j}^{[g]}(t)\right) x
$$

Then, thanks to (20), it follows that

$$
\mathcal{L} V(x, s, t)<-\left(\sum_{k=1}^{M} \varphi_{g k} q_{s}^{[k]}(t)^{\prime}\right) x
$$

Since $\left(q_{s}^{[k]}\right)^{\prime} x \geq\left(q_{s}^{[k]}\right)^{\prime} x, \forall k$ and $\sum_{k=1}^{m} \varphi_{g k}=0$, it can be shown that

$$
\begin{aligned}
\mathcal{L} V(x, s, t) & <-\left(\sum_{k \neq g} \varphi_{g k}\left(q_{s}^{[k]}\right)^{\prime}\right) x-\varphi_{g g}\left(q_{s}^{[g]}\right)^{\prime} x \leq \\
& \leq-\left(\sum_{k \neq g} \varphi_{g k}\left(q_{s}^{[g]}\right)^{\prime}\right) x-\varphi_{g g}\left(q_{s}^{[g]}\right)^{\prime} x= \\
& =-\sum_{k=1}^{M} \varphi_{g k}\left(q_{s}^{[g]}\right)^{\prime} x=0
\end{aligned}
$$

Since $\mathcal{L} V(x, s)<0$, the system under the action of (21) is mean stable (see [17]), and, moreover, using Dynkin's formula we obtain

$$
\begin{aligned}
J\left(\gamma^{*}, x_{0}\right) & =E\left[c_{\sigma\left(t_{f}\right)}^{\prime} x\left(t_{f}\right)\right] \\
& =E\left[\min _{r} q_{\sigma\left(t_{f}\right)}^{[r]}\left(t_{f}\right)^{\prime} x\left(t_{f}\right)\right] \\
& <E\left[\min _{r} q_{\sigma(0)}^{[r]}(0)^{\prime} x_{0}\right] \\
& \leq \min _{r} E\left[q_{\sigma(0)}^{[r]}(0)^{\prime} x_{0}\right]=\min _{r} \sum_{i=1}^{N} \bar{\pi}_{i} q_{i}^{[r]}(0)^{\prime} x_{0}
\end{aligned}
$$

It goes without saying that the upper bound can be minimized by looking at the best $\Phi \in \mathcal{G}_{m}$. For $t_{f} \rightarrow \infty$ results on optimal control (with integral action) and mean stabilization have been given in [23], [22]. 
Taking again the propagation of $E[x(t)]$ with $s_{i}(t)=E[x(t) \mid \sigma(t)=i] \bar{\pi}_{i}$ the switched system

$$
\dot{s}=\left(\bar{M}+\bar{\Lambda}^{[\gamma]}\right) s, \quad s(0)=\bar{s}_{0}
$$

is obtained, where $\bar{M}$ and $\bar{s}_{0}$ have been already defined and

$$
\begin{aligned}
\bar{\Lambda}^{[j]} & =\left[\begin{array}{cccc}
\Lambda_{1}^{[j]} & 0 & \cdots & 0 \\
0 & \Lambda_{2}^{[j]} & \cdots & 0 \\
\vdots & \vdots & \ddots & \vdots \\
0 & 0 & \cdots & \Lambda_{N}^{[j]}
\end{array}\right] \\
\bar{s}_{0} & =\bar{\pi} \otimes x_{0} .
\end{aligned}
$$

Moreover, if the vectors $q_{i}^{j}$ are grouped as follows $\left(q^{j}\right)^{\prime}=\left[\left(q_{1}^{j}\right)^{\prime}\left(q_{2}^{j}\right)^{\prime} \ldots\left(q_{N}^{j}\right)^{\prime}\right]$, equations (20) can be compactly rewritten as

$$
0=\dot{q}^{[j]}(t)^{\prime}+q^{[j]}(t)^{\prime} \bar{A}^{[j]}+\sum_{r=1}^{m} \phi_{j r} q^{[r]}(t)^{\prime}, \quad q^{[j]}\left(t_{f}\right)=\bar{c}, \quad \forall j
$$

with $\bar{c}$ already defined. Therefore, letting $\bar{A}^{[j]}:=\bar{M}+\bar{\Lambda}^{[j]}$, following theorem holds, see Corollary 1 in [27].

Theorem 5: Take any Metzler matrix $\Phi=\left[\phi_{i j}\right] \in \mathcal{G}_{m}$ and consider the solutions $q^{[j]}(t)$, $j=1,2, \cdots, m$ satisfying (23). The control law

$$
\tilde{\gamma}^{*}(t)=g(t, s)=\arg \min _{r} q^{[r]}(t)^{\prime} s(t)
$$

is such that $J\left(\tilde{\gamma}^{*}, x_{0}\right)<\min _{r} q^{[r]}(0)^{\prime} s(0)=\min _{r} \sum_{i=1}^{N} \bar{\pi}_{i} q_{i}^{[r]}(0)^{\prime} x_{0}$.

Remark 8: Note that the upper bounds obtained in Theorems 4 and 5 do coincide. The deterministic control law $\tilde{\gamma}^{*}(t)$ in Theorem 5 can be also written as $\tilde{\gamma}^{*}(t)=\arg \min _{r} E\left[q_{\sigma(t)}^{[r]}(t)^{\prime} x(t)\right]$, so marking the difference with respect to the stochastic control law in Theorem 4 . Note also that $\tilde{\gamma}^{*}(t)$ is not practically implementable since knowledge of $s(t)$ is required, whereas $\gamma^{*}(t)$ can be implemented in the original stochastic system if one possesses on-line information on the stochastic process $\sigma$ and the state variable $x$ (full information). On the other hand, one can think of replacing $s_{i}$ with $\bar{\pi}_{i} x$ in the control law $\tilde{\gamma}^{*}(t)$, where $x$ is the current state, so 
yielding a sort of average strategy

$$
\gamma^{a v}(t)=\arg \min _{r} \sum_{i=1}^{N} \bar{\pi}_{i} q_{i}^{[r]}(t)^{\prime} x(t)
$$

or consider the worst $\sigma(t)$ yielding the worst case strategy

$$
\gamma^{w c}(t)=\arg \min _{r} \max _{i} q_{i}^{[r]}(t)^{\prime} x(t)
$$

However, tot he best of the authors' knowledge, although implementable, these pure statefeedback laws have no guaranteed performance nor are they guaranteed to be stabilizing for $t_{f} \rightarrow \infty$. The definition of mode independent state-feedback strategies for stabilization and or optimal control problems is still an open issue worth of further investigation. The estimation of $\sigma$ from available measurements calls for the theory of hidden Markov chains, and references can be made to the available literature, see e.g [24]. Preliminary promising results, based on minimax theory, are also available, see [25], [22].

Remark 9: Equations (20) are those used for the computation of $E[x]$ for a Markov Jump System obtained considering $\gamma(t)$ as a Markovian process, independent of $\sigma(t)$, generated by $\Phi \in \mathcal{G}_{m}$. To be precise, we can define an extended Markovian process $\sigma_{e}(t)$ taking values in $\mathcal{N} \times \mathcal{M}$, and rewrite system (18) as follows

$$
\dot{x}=F_{\sigma_{e}} x
$$

where $F_{k}=A_{i}^{[k-(i-1) M]}$ for $i=1,2, \ldots, N$ and $k=(i-1) M+1, \ldots, i M$. This system is a positive Markov jump system. The stationary probability distribution of this extended positive Markov jump system is $\hat{\pi}=\bar{\pi} \otimes \bar{f}$, where $\bar{f}$ be the stationary probability measure associated with $\Phi$, i.e. $\Phi \bar{f}=0$. The rate transition matrix of $\sigma_{e}$ is $\Pi_{e}=\Pi \oplus \Phi=\Pi \otimes I+I \otimes \Phi$. Letting $\zeta_{k}(t)=E\left[x(t) \mid \sigma_{e}(t)=k\right] \hat{\pi}_{k}$ the mean evolution of the state variables can be described through the deterministic positive system

$$
\dot{\zeta}(t)=\hat{F} \zeta(t)
$$


where

$$
\hat{F}=\left[\begin{array}{cccc}
F_{1} & 0 & \cdots & 0 \\
0 & F_{2} & \cdots & 0 \\
\vdots & \vdots & \ddots & \vdots \\
0 & 0 & \cdots & F_{N M}
\end{array}\right]+\Pi_{e} \otimes I_{n}
$$

In the same vein, the cost $E\left[c_{\sigma\left(t_{f}\right)}^{\prime} x\left(t_{f}\right)\right]$ can be rewritten as $\tilde{c}^{\prime} \zeta\left(t_{f}\right)$, where

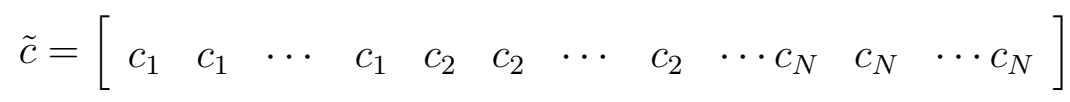

In conclusion, take any Metzler matrix $\Phi=\left[\phi_{i j}\right] \in \mathcal{G}_{m}$ and let $\gamma(t)$ be the stochastic process (independent of $\sigma(t))$ associated with it. Then

$$
E\left[c_{\sigma(t)}^{\prime} x\left(t_{f}\right)\right]=q(0)^{\prime} \zeta(0)
$$

where $q$ satisfies

$$
\dot{q}(t)^{\prime}+q(t)^{\prime} \hat{F}=0, \quad q\left(t_{f}\right)^{\prime}=\tilde{c}^{\prime}
$$

and $\zeta(0)=\hat{\pi} \otimes x_{0}$. Notice that both $q(0)$ and $\hat{\pi}$ depend on $\Phi$. Therefore, the cost can be optimized as a function of $\Phi \in \mathcal{G}_{m}$.

B. $\gamma(t)$ as a stochastic process function of $\sigma(t)$ only

We now consider the case where $\gamma(t)$ is a surjective function of $\sigma(t)$, say $\gamma=b(\sigma)$. In this case $\sigma$ and $\gamma$ are no longer independent and the system

$$
\dot{x}=\left(M_{\sigma}+\Lambda_{\sigma}^{[b(\sigma)]}\right) x
$$

is a positive Markov Jump Linear systems with $\Pi$ as infinitesimal transition probability matrix. Taking the propagation of the expectations $s_{i}(t)=E[x(t) \mid \sigma(t)=i]$, and letting $A_{i}^{[j]}=M_{i}+\Lambda_{i}^{[j]}$, it follows

$$
\dot{s}=\left[\begin{array}{cccc}
A_{1}^{[b(1)]}+\pi_{11} I_{n} & \pi_{21} I_{n} & \cdots & \pi_{N 1} I_{n} \\
\pi_{12} I_{n} & A_{2}^{[b(2)]}+\pi_{21} I_{n} & \cdots & \pi_{N 2} I_{n} \\
\vdots & \vdots & \ddots & \vdots \\
\pi_{1 N} I_{N} & \pi_{2 N} I_{N} & \cdots & A_{N}^{[b(N)]}+\pi_{N N} I_{n}
\end{array}\right] s
$$


The above system is a deterministic positive system, so that, for a given map $g(\sigma)$, the cost $E\left[c_{\sigma(t)}^{\prime} x\left(t_{f}\right)\right]=\bar{c}^{\prime} s\left(t_{f}\right)$ can be easily computed. Therefore, the following result can be stated.

Theorem 6: Consider the positive Markov Jump Linear system system (28) and the differential equation

$$
0=\dot{q}_{i}^{[g(i)]}(t)^{\prime}+q_{i}^{[b(i)]}(t)^{\prime} A_{i}^{[b(i)]}+\sum_{k=1}^{N} \pi_{i k} q_{k}^{[b(k)]}(t)^{\prime}, \quad q_{i}^{[b(i)]}\left(t_{f}\right)=c_{i},
$$

Then,

$$
E\left[c_{\sigma(t)}^{\prime} x\left(t_{f}\right)\right]=\bar{c}^{\prime} s\left(t_{f}\right)=\sum_{i=1}^{N} \bar{\pi}_{i} q_{i}^{[b(i)]}(0)^{\prime} x(0)
$$

Proof: The proof follows a rationale similar to that of Theorem 4, by considering the evolution of the stochastic Lyapunov function $V(x, s, t)=q_{s}^{[g(s)]}(t)^{\prime} x$. It is therefore omitted.

Again, one can choose, among the finite number of maps $g(\sigma)$, the one that gives the best cost. For $t_{f} \rightarrow \infty$ we have results on optimal control (with an integral action) and stabilization in the sense of Mean Stability, see [1], [22].

\section{Infinite horizon}

The infinite horizon optimal control problem calls for the notion of stability. As such, since (1) is a positive stochastic system and a linear integral cost is considered (for $t \rightarrow \infty$ ), the notion of stability that naturally arises is the so-called mean stability, equivalent to the asymptotic convergence of the expectation of the state variable to zero. As shown in [1] this is also equivalent to the convergence to zero of the first moment $(E[\|x\|])$ of the state vector, for any initial state (or just for one strictly positive initial state thanks to the monotonicity property of positive systems). A necessary and sufficient condition for mean stability of (18) with $\gamma(t)=\bar{\gamma} \in \mathcal{M}$ constant is the asymptotic stability of the extended deterministic system (22). Turning to optimal control, in order to be sure that there exists a solution of the optimal control problem

$$
J_{\infty}\left(\gamma, x_{0}\right)=E\left[\int_{0}^{\infty} d_{\sigma(t)}^{\prime} x(t) d t\right]
$$

with $d_{i}$ positive vectors, $i \in \mathcal{N}$, the system should possess the property of mean stabilizability, that is the existence of a switching law $\gamma(t)$ such that the closed-loop system is stable in 
the mean sense.

The stabilization problem, even for deterministic positive systems, is a formidable task for dimensions greater than 2, see the recent paper [5].

A sufficient condition for mean stabilizability, that also provides an upper bound to the cost is given by looking at the infinite-time version of Theorem 4 , hinging on the so-called co-positive dual Lyapunov-Metzler inequalities

$$
\left(q_{i}^{[k]}\right)^{\prime} A_{i}^{[k]}+\sum_{j=1}^{N} \pi_{i j}\left(q_{j}^{[k]}\right)^{\prime}+\sum_{s=1}^{m} \phi_{k s}\left(q_{i}^{[s]}\right)^{\prime}+d_{i}<0
$$

with $i \in \mathcal{N}, k \in \mathcal{M}$ and $\Phi \in \mathcal{G}_{m}$. If there exist nonnegative vectors $q_{i}^{[k]}$ and nonnegative numbers $\phi_{k s}, k \neq s$ satisfying (31), then the switching law

$$
\gamma=\underset{k}{\operatorname{argmin}}\left(q_{\sigma}^{[k]}\right)^{\prime} x
$$

is stabilizing in the mean sense and such that $J_{\infty}\left(\gamma, x_{0}\right)<\min _{k} \sum_{i=1}^{N} \bar{\pi}_{i}\left(q_{i}^{[k]}\right)^{\prime} x_{0}$, see Theorem 9 in [23]. If this is the case, one can optimize the upper bound with respect to the free parameters $\phi_{r s}$. Note that the above control requires knowledge of $\sigma$. The same upper bound is provided by the deterministic feedback law applied to the extended system (6)

$$
\tilde{\gamma}=\underset{k}{\operatorname{argmin}}\left(q^{[k]}\right)^{\prime} s
$$

where vector $q^{[j]}$ is formed by stacking all vectors $q_{i}^{[j]}, i \in \mathcal{N}$ in a sole vector. This result is the extension to the infinite horizon case of Theorem 5. As already observed in Section III-A, this last control law cannot be applied to the original system and one may try to substitute it with $\gamma^{a v}=\operatorname{argmin}_{k} \sum_{i=1}^{N} \bar{\pi}_{i} q_{i}^{[k]} x$, or $\gamma^{w s}=\operatorname{argmin}_{k} \max _{i} p_{i}^{[k]} x$, without any guarantee of stability, unless the original system is stable under arbitrary switching, as occurs in the example presented next.

When $\gamma(t)$ is chosen as a static map of $\sigma(t)$, i.e. $\gamma(t)=b(\sigma(t))$, where $b(\sigma)$, one obtains the extension of Theorem 5 to the infinite horizon case. To be precise, with $\gamma(t)=g(\sigma(t))$, the cost obtained is

$$
J_{\infty}\left(\gamma, x_{0}\right)=\sum_{i=1}^{N} \bar{\pi}_{i}\left(q_{i}^{[b(i)]}\right)^{\prime} x(0)
$$


where

$$
0=\left(q_{i}^{[b(i)]}\right)^{\prime} A_{i}^{[b(i)]}+\sum_{k=1}^{N} \pi_{i k}\left(q_{k}^{[b(k)]}\right)^{\prime}+d_{i}^{\prime}
$$

and of course one can look at the best map optimizing the cost.

\section{Diagonally switching deterministic systems}

In this session, we discuss the stabilization and optimal control problem of a class of deterministic positive switched systems, that will describe the epidemiological system considered in the final section. This class is characterized by the fact that the switching control affects only the diagonal elements of the dynamic matrix. To be precise we consider systems described by

$$
\left.\dot{x}(t)=A^{[\gamma]} x(t)=\left(\Pi^{\prime}+\Lambda^{[\gamma]}\right]\right) x(t)
$$

where $\Pi$ is a Metzler matrix and $\Lambda^{[i]}, i \in \mathcal{M}=\{1,2, \ldots, m\}$ are diagonal matrices with components $\lambda_{j}^{i}, j \in \mathcal{M}=\{1,2, \ldots, N\}$, where $N$ is the dimension of the system. Without any loss of generality we can assume that $\Pi \mathbf{1}=0$.

It is easy to see that to system (34) we can associate the scalar stochastic system

$$
\dot{\xi}(t)=\lambda_{\sigma(t)}^{[\gamma(t)]} \xi(t)
$$

where $\sigma(t)$, taking values in $\mathcal{N}=\{1,2, \ldots, N\}$, is a form process governed by a Markov

chain with infinitesimal transition probability matrix $\Pi$. Denote as $\bar{\pi}_{i}$ the components of the stationary probability associated with $\Pi$, i.e. $\Pi^{\prime} \bar{\pi}=0$, the variables $x_{i}(t)$ of the original system can be seen as (the simple proof is omitted)

$$
x_{i}(t)=E[\xi(t) \mid \sigma(t)=i] \bar{\pi}_{i}, \quad \sum_{i=1}^{N} x_{i}(t)=E[\xi(t)]
$$

System (35) is a dual switching system studied in Section III.

\section{E. Stability and stabilization of system (34)}

Recalling the definition of mean stability of a Markovian system, [1], [22], the following one-to-one relation between (34) and (35) holds. 
Theorem 7: The system (34) is stable under arbitrary switching if and only if the system (35) is mean-stable under arbitrary switching. The system (34) is stabilizable if and only if system (35) is mean-stabilizable.

Observe that the evolution formula for $\xi(t)$ can be explicitly written as

$$
\xi(t)=e^{\int_{0}^{t} \lambda_{\sigma(\tau)}^{[\gamma(\tau)]}} d \tau(0)
$$

Therefore, it is easy to study the convergence to zero of the state $\xi(t)$ with probability one (almost sure stability). Indeed, for a constant $\gamma$, the system (35) is almost sure stable if and only if there exists $T>0$ such that $E[\log (\Phi(T, 0))]<0,[10]$, and this happens if and only if $\sum_{i=1}^{n} \bar{\pi}_{i} \lambda_{i}^{[\gamma]}<0$. Therefore system (35) is almost surely stable under arbitrary switching if and only if $\max _{r} \sum_{i=1}^{n} \bar{\pi}_{i} \lambda_{i}^{[r]}<0$. Furthermore, it is almost surely stabilizable if and only if $\min _{r} \sum_{i=1}^{n} \bar{\pi}_{i} \lambda_{i}^{[r]}<0$. It is however well known that almost sure stability is a weaker stability notion than mean stability. Therefore, the following result can be stated (the proof is trivial).

Theorem 8: (i) System (34) is stable under arbitrary (deterministic) switching only if $\max _{r} \sum_{i=1}^{n} \bar{\pi}_{i} \lambda_{i}^{[r]}<0$. (ii) System (34) is stabilizable via a (deterministic) switching law only if $\min _{r} \sum_{i=1}^{n} \bar{\pi}_{i} \lambda_{i}^{[r]}<0$.

Again, from the evolution formula we have

$$
\int^{\int_{0}^{t_{f}} \lambda_{\sigma(\tau)}^{[\tilde{\gamma}(\tau)]} d \tau} \xi(0) \leq \xi(t) \leq e \int_{0}^{t_{f}} \lambda_{\sigma(\tau)}^{[\hat{\gamma}(\tau)]} d \tau
$$

where

$$
\left.\left.\tilde{\gamma}(t)=\tilde{b}(\sigma(t))=\arg \min _{r} \lambda_{\sigma(t)}^{[} r\right], \quad \hat{\gamma}(t)=\hat{b}(\sigma(t))=\arg \max _{r} \lambda_{\sigma(t)}^{[} r\right]
$$

The system (35) under $\tilde{\gamma}$ is the "best" system described by $\dot{\tilde{\xi}}=\lambda_{\sigma}^{[\tilde{b}(\sigma)]} \tilde{\xi}$ whereas the system (35) under $\hat{\gamma}$ is the "worst" system described by $\dot{\hat{\xi}}=\lambda_{\sigma}^{[\hat{b}(\sigma)]} \hat{\xi}$. Correspondingly, the dynamics 
of $\tilde{x}_{i}(t)=E[\tilde{\xi}(t) \mid \sigma(t)=i] \bar{\pi}_{i}$ is given by

$$
\begin{aligned}
\dot{\tilde{x}} & =\tilde{A} \tilde{x}, \quad \tilde{A}=\Pi^{\prime}+\tilde{\Lambda} \\
\tilde{\Lambda} & =\left[\begin{array}{cccc}
\lambda_{1}^{[\tilde{b}(1)]} & 0 & 0 & 0 \\
0 & \lambda_{2}^{[\tilde{b}(2)]} & 0 & 0 \\
\vdots & \vdots & \ddots & \vdots \\
0 & 0 & \cdots & \lambda_{N}^{[\tilde{b}(N)]} I_{n}
\end{array}\right]
\end{aligned}
$$

whereas the dynamics of $\hat{x}_{i}(t)=E[\hat{\xi}(t) \mid \sigma(t)=i] \bar{\pi}_{i}$ is given by

$$
\begin{aligned}
\dot{\hat{x}} & =\hat{A} \hat{x}, \quad \hat{A}=\Pi^{\prime}+\hat{\Lambda} \\
\hat{\Lambda} & =\left[\begin{array}{cccc}
\lambda_{1}^{[\hat{b}(1)]} & 0 & 0 & 0 \\
0 & \lambda_{2}^{[\hat{b}(2)]} & 0 & 0 \\
\vdots & \vdots & \ddots & \vdots \\
0 & 0 & \cdots & \lambda_{N}^{[\hat{b}(N)]} I_{n}
\end{array}\right]
\end{aligned}
$$

We are now in the position to prove

Theorem 9: (i) The system (34) is stable under arbitrary switching if the matrix $\hat{A}$ is Hurwitz. (ii) The system (34) is stabilizable via a switching law only if $\tilde{A}$ is Hurwitz.

Proof: (i) Assume that $\hat{A}$ is Hurwitz and take the co-positive linear Lyapunov function $V(x)=\hat{c}^{\prime} x$ where the strictly positive vector $\hat{c}$ is such that $\hat{c}^{\prime} \hat{A}=\hat{c}^{\prime}\left(\Pi^{\prime}+\hat{\Lambda}\right) \ll 0$. It turns out that $\dot{V}(x)=\hat{c}^{\prime} A_{\gamma} x=\hat{c}^{\prime}\left(\Pi^{\prime}+\Lambda^{[\gamma]}\right) x \leq \hat{c}^{\prime}\left(\Pi^{\prime}+\hat{\Lambda}\right)<0$ so that system (34) is stable under arbitrary switching.

(ii) Assume that system (34) is stabilizable and $\tilde{A}$ not Hurwitz. Then there exists $\gamma(t)=$ $\gamma^{*}(t)$ such that system $(34)$ is asymptotically stable and a positive vector $\tilde{c}$ such that $\tilde{c}^{\prime} \tilde{A}=$ $\tilde{c}^{\prime}\left(\Pi^{\prime}+\Lambda^{\left[\gamma^{*}\right]}\right)=\mu \tilde{c}$ with $\mu>0$. Take the function $V(x)=\tilde{c}^{\prime} x$. It turns out that $\dot{V}(x)=$ $\tilde{c}^{\prime} A_{\gamma^{*}} x=\tilde{c}^{\prime}\left(\Pi^{\prime}+\Lambda^{\left[\gamma^{*}\right]}\right) x \geq c^{\prime}\left(\Pi^{\prime}+\tilde{\Lambda}\right)=\mu(\tilde{c})^{\prime} x>0$ so that system (34) with $\gamma(t)=\gamma^{*}(t)$ is not stable, a contradiction.

The conditions in the theorem above are not necessary and sufficient, even for second order switched systems, as the following example demonstrates. Take for instance system 


$$
A^{[1]}=\left[\begin{array}{cc}
-2 & 1 \\
1 & -0.9
\end{array}\right], \quad A^{[2]}=\left[\begin{array}{cc}
-0.9 & 1 \\
1 & -2
\end{array}\right]
$$

The underlying infinitesimal Markov transition matrix is then

$$
\Pi=\left[\begin{array}{cc}
-1 & 1 \\
1 & -1
\end{array}\right]
$$

and

$$
\Lambda^{[1]}=\left[\begin{array}{cc}
-1 & 0 \\
0 & 0.1
\end{array}\right], \quad \Lambda^{[2]}=\left[\begin{array}{cc}
0.1 & 0 \\
0 & -1
\end{array}\right]
$$

It is clear that the two matrices are Hurwitz. It is easy to check that they do not share any common linear co-positive Lyapunov function $V(x)=c^{\prime} x$ with $c \gg 0$ or $V(x)=\max _{i} \frac{x_{i}}{d_{i}}$ with $d \gg 0$. However, they share a quadratic Lyapunov function $V(x)=x^{\prime} P x$, with $P$ positive definite, (for example)

$$
P=\left[\begin{array}{ll}
2 & 1 \\
1 & 2
\end{array}\right]
$$

As such the switched system is stable under arbitrary switching even though

$$
\hat{A}=\left[\begin{array}{cc}
-0.9 & 1 \\
1 & -0.9
\end{array}\right]
$$

is unstable. Other simple examples can be found for the stabilizability problem.

Since $\xi(t)>0$, it follows that

$$
\tilde{\gamma}(t)=\tilde{b}(\sigma(t))=\arg \min _{r} \lambda_{[\sigma(t)]}^{r}=\arg \min _{r} \lambda_{\sigma(t)}^{[r]} \xi(t)
$$

Therefore, mimicking the best (non implementable) strategy $\tilde{\gamma}(t)$ above, one can devise an implementable state-feedback average strategy

$$
\gamma^{a v}(t)=\arg \min _{r} \sum_{i=1}^{n} \lambda_{i}^{[r]} x_{i}(t)
$$

Of course this strategy is very simple but in general is not stabilizing in the mean sense, unless $\Pi^{\prime}+\hat{\Lambda}$ is stable (so implying that any switching strategy does not destroy mean 
stability).

F. Optimal control for system (34)

We now associate to system (34) the cost function

$$
J_{\infty}\left(\gamma, x_{0}\right)=\int_{0}^{\infty} \mathbf{1}^{\prime} x(t) d t
$$

which corresponds to

$$
J_{\infty}\left(\gamma, x_{0}\right)=E\left[\int_{0}^{\infty} \xi(t) d t\right]
$$

for the dual switching system (35). The minimization of $J_{\infty}\left(\gamma, x_{0}\right)$ can be performed in open loop by considering the relaxation discussed in Remark 7, exploiting the convexity of the

functional $E\left[\int_{0}^{t_{f}} \xi(t) d t\right]$ with respect to the control variables, see Section II-A, and taking $t_{f}$ sufficiently large. Suboptimal feedback control laws can be devised following the discussion in Section III.

\section{ExAmple}

\section{A. description of the system and the problem}

This example is taken from [20] and concerns the epidemiological model of a population divided into $n$ groups. Each group is divided into two classes, i.e. $I_{i}(t)$ infectives and $S_{i}(t)$ susceptibles. Under the assumption that the total number $I_{i}(t)+S_{i}(t)=N_{i}$ is constant and letting $x_{i}(t)=I_{i}(t) / N_{i}$ one can write, for $i=1,2, \cdots, n$ :

$$
\dot{x}_{i}(t)=\left(1-x_{i}(t)\right) \sum_{j=1}^{N} \frac{\beta_{i j} N_{j}}{N_{i}} x_{j}(t)-\left(\eta_{i}+\mu_{i}\right) x_{i}(t)
$$

where $\beta_{i j}$ is the rate at which susceptibles in group $i$ are infected by infectives in group $j$, $\eta_{i}$ is the rate at which an infective individual in group $i$ is cured and $\mu_{i}$ the birth and death rates in group $i$ (assumed to be equal since the number of the total population in the same group is constant). Note that the set $0 \leq x_{i} \leq 1, i=1,2, \cdots, 4$, is positively invariant.

We now assume that, for each group $i, m$ different cures are possible, so that the rate $\eta_{i}$ depends on an additional index, say $\gamma=\{1,2, \cdots, m\}$, that represents the switching signal that orchestrates the different therapies for each group $i$. Therefore, we replace $\eta_{i}$ in (44) 
with $\eta_{i}^{[\gamma]}$. The addition of the therapy scheduling preserves the positive invariance property of the set $0 \leq x_{i} \leq 1, i=1,2, \cdots, 4$. We also make the simplifying assumption that the change of therapies does not affect the rates $\beta_{i j}$. Finally, we linearize the system around the disease free equilibrium $x=0$. The linearized system is then given by (35), i.e.

$$
\dot{x}=\left(\Pi^{\prime}+\Lambda^{[\gamma]}\right) x
$$

where $\Pi$ is a Metzler matrix with $\Pi \mathbf{1}=0$ and entries $\pi_{i j}=\beta_{i j} N_{j} / N_{i}, i \neq j$ and $\Lambda^{[1]}, \Lambda^{[2]}, \cdots, \Lambda^{[m]}$ are diagonal matrices. The entries of $\Lambda^{[\gamma]}$ are denoted by $\lambda_{i}^{[\gamma]}$ and are as follows: $\lambda_{i}^{[\gamma]}=$ $-\eta^{[\gamma]}-\mu_{i}+\sum_{j \neq i} \beta_{j i} N_{i} / N_{j}$. We can define the associated embedded system as

$$
\dot{x}=\left(\Pi^{\prime}+\Lambda(u)\right) x
$$

where the vector $u=\left[u_{1}, u_{2}, \cdots u_{m}\right]^{\prime}$ lives in the polytope $\sum_{i=1}^{m} u_{i}=1, u_{i} \geq 0$ and $\Lambda(u)=$ $\sum_{i=1}^{m} \Lambda_{i} u_{i} . \Lambda(u)$ is convex with respect to $u$, so that the result of Section II can be used to conclude that, given a final time $t_{f}$, the scalar cost functional $J\left(u, x_{0}\right)=f\left(x\left(t_{f}\right)\right)$ is a convex functional of $u \in \mathcal{U}_{s}$ provided that $f$ is a monotonically increasing function. In the following example we consider the infinite time optimal control problem

$$
J\left(u, x_{0}\right)=\int_{0}^{\infty} \mathbf{1}_{n}^{\prime} x(t) d t
$$

It goes without saying that in order for the problem to make sense, the system (45) should be stabilizable.

1) Optimal control: Consider a switched linear positive system in which the control switching signal $\gamma$ affects only the diagonal elements of the dynamic matrix. As such, the optimal (time-driven) strategy can be found by exploiting the convexity of the problem, [9], $[2]$.

2) Lyapunov-Metzler inequalities: A sufficient condition for stabilizability, also allowing a computation of an upper bound to the optimal cost, is given by looking at the so-called co-positive Lyapunov-Metzler inequalities, see (20)

$$
\left(q^{[r]}\right)^{\prime} A^{[r]}+\sum_{j \neq i} \phi_{r j}\left(\left(q^{[j]}\right)^{\prime}-\left(q^{[r]}\right)^{\prime}\right)+\mathbf{1}_{n}^{\prime}<0, \quad i=1,2, \cdots, m
$$


If there exist nonnegative vectors $q^{[r]}, r=1,2, \cdots, m$, and nonnegative numbers $\phi_{r j}, j \neq$ $r$ satisfying (48), then the switching law $\gamma=\operatorname{argmin}_{r}\left(q^{[r]}\right)^{\prime} x$ is stabilizing and such that $J\left(\gamma, x_{0}\right)<\min _{r}\left(q^{[r]}\right)^{\prime} x_{0}$. If this is the case, one can optimize the upper bound with respect to the free positive parameters $\phi_{r j}, r \neq j$.

3) Average and worst-case strategies: The system can be rewritten as a dual switching scalar system

$$
\dot{\xi}=\lambda_{\sigma}^{[\gamma]} \xi
$$

where $\sigma$ is a form process with infinitesimal transition matrix $\Pi$. Accordingly the cost can be written as: $J\left(\gamma, x_{0}\right)=E\left[\int_{0}^{\infty} \xi(t) d t\right.$. The optimal control is then given by the $\sigma / \gamma$-map:

$$
\gamma^{o p t}(t)=\arg \min _{r} \lambda_{[\sigma(t)]}^{r}
$$

Since $\sigma$ is an unavailable switching signal, one can use the average strategy as introduced in (41).

\section{B. Simulation results}

Consider system (44) and its linearized version (45) with

$$
\begin{aligned}
\Pi^{\prime} & =\left[\begin{array}{cccc}
-1.9462 & 0.6324 & 0.9575 & 0.9572 \\
0.9058 & -1.4578 & 0.9649 & 0.4854 \\
0.1270 & 0.2785 & -2.8930 & 0.8003 \\
0.9134 & 0.5469 & 0.9706 & -2.2429
\end{array}\right] \\
\Lambda^{[1]} & =\operatorname{diag}\{-1.6811,-0.9587,-3.0005,-0.1098\} \\
\Lambda^{[2]} & =\operatorname{diag}\{-0.0199,-1.4265,-2.1732,-2.2430\}
\end{aligned}
$$

The task is to find the optimal control that minimizes

$$
J\left(u, x_{0}\right)=\int_{0}^{\infty} \sum_{i=1}^{4} x_{i}(t) d t
$$

with the initial state given by $x_{0}=\left[\begin{array}{lllll}0.05 & 0.15 & 0.25 & 0.35\end{array}\right]^{\prime}$.

Letting $c^{\prime}=\left[\begin{array}{llll}0 & 0 & 0 & 0\end{array}\right]$, and according to (47) we can tackle the problem of minimizing 
$J\left(u, x_{0}\right)=\lim _{t_{f} \rightarrow \infty} c^{\prime} \xi\left(t_{f}\right)$ under the system dynamics given by $\dot{\xi}=A(u) \xi$, where

$$
A(u)=\left[\begin{array}{cc}
\Pi^{\prime}+\Lambda(u) & 0 \\
\mathbf{1}_{4}^{\prime} & 0
\end{array}\right]
$$

and $\Lambda(u)=\Lambda^{[1]} u_{1}+\Lambda^{[2]}\left(1-u_{1}\right)$

Notice, recall (37), (38), that (element-wise)

$$
\Pi^{\prime}+\tilde{\Lambda}<\Pi^{\prime}+\Lambda^{[\gamma]}<\Pi^{\prime}+\hat{\Lambda}
$$

A simple computation shows that $\Pi^{\prime}+\hat{\Lambda}$ is Hurwitz stable so that we can conclude that the system is stable under arbitrary switching and the best stochastic switching control signal is the one that select $\gamma$ according to the Markov process $\sigma$ with transition probability matrix $\Pi$ as in $(36)$

$$
\tilde{\gamma}^{s}(t)=\operatorname{argmin}_{k}\left(\lambda^{[k]}\right)_{\sigma(t)}
$$

which results in $\gamma(t)=1$, if $\sigma(t)=1$ or $\sigma(t)=3$ and $\gamma(t)=2$ if $\sigma(t)=2$ or $\sigma(t)=4$. Of course this switching law is not implementable since $\sigma$ is an hidden signal whose only meaning is to interpret the state components $x_{i}(t)$ as the expectation of an hidden stochastic process. In any case with this map, say $\gamma=b(\sigma)$, one would obtain $J\left(b, x_{0}\right)=-\mathbf{1}_{4}^{\prime}\left(\Pi^{\prime}+\tilde{\Lambda}^{-1}\right) x_{0}=$ 0.4, and this is a lower bound to the cost for any possible strategies. Therefore we have implemented the average strategy, see (41)

$$
\gamma^{a v}(t)=\arg \min _{k} \sum_{i=1}^{4} \lambda_{i}^{[k]} x_{i}
$$

With this strategy the cost is $J\left(\gamma^{a v}, x_{0}\right)=0.58$.

Now, we consider constant $u$. Notice that both $A^{[1]}=\Pi^{\prime}+\Lambda^{[1]}$ and $A^{[2]}=\Pi^{\prime}+\Lambda^{[2]}$ are Hurwitz stable, so that the best value of the cost for constant $u$ in the vertices can be easily found $\min _{i} q_{i}^{\prime} x_{0}$ where $q_{i}=-\mathbf{1}_{4}^{\prime}\left(\Lambda^{[i]}+\Pi^{\prime}\right)^{-1}, i=1,2$. It turns out that $J\left(1, x_{0}\right)=$ $\min _{i} q_{i}^{\prime} x_{0}=0.622$. Notice that by taking a constant $u$ strictly inside the polytope, the best constant control $\bar{u}$ is $\bar{u}_{1}=0.26, \bar{u}_{2}=0.74$, that corresponds to a sliding mode for the switching system. With this control the cost is $J\left(\bar{u}, x_{0}\right)=0.601$.

One can also optimize the values of the parameters $\phi_{i j}$ in order to minimize the upper 
bound of the cost given by the co-positive Lyapunov-Metzler inequalities (48). Taking $\phi_{12}=$

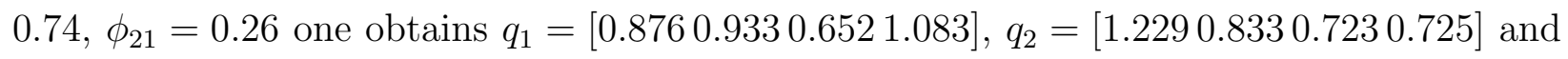
applying the associated sub-optimal switching strategy one obtains a cost again equal to 0.58. The (state-feedback) control law generates a sort of periodic behaviour in that the control switches from $u_{1}=0$ to $u_{1}=1$ in a periodic fashion.

We have computed the optimal control $u^{o}$ numerically, with a time horizon of 20 seconds, and 0.1 second discretization, using an analytical gradient, and the interior point algorithm in the Matlab function, fmincon. Surprisingly, in this particular case, the optimal cost for the linearized system, $J\left(u^{o}, x_{0}\right)=0.58$, is equal to the cost obtained by the sub-optimal switching strategy and to the average and worst-case strategies.

As for the concave-convex mixed strategy, see (15), (16), the results show that the worst initial state is $x_{0}=\alpha[0.2060 .1631,0.00430 .126]^{\prime}$, where $\alpha$ is any positive scalar. Notice that since the system is linear the cost is also linear with respect to $x_{0}$ and hence only the direction is important. The associated cost is $0.4321 \alpha$.

When applied to the nonlinear system, (44), the best constant control within the vertices is with $u_{1}=0$ and the associated cost is 0.411 . The best constant control within the polytope is $u_{1}=0.26, u_{2}=0.74$ and the associated cost is 0.403 . The switching strategy based on the LM inequalities provides a cost equal to 0.3922 . If we compute the optimal control worked out for the linearized system, the cost is again equal (up to numerical errors) to the cost due to the switching strategy based on the co-positive Lyapunov-Metzler inequalities, whereas the myopic strategy results in a cost equal to 0.4314. Finally, the min-max optimal strategy with $x_{0}=[0.2060 .16310 .00430 .126]^{\prime}$ has been applied to the nonlinear system, giving a cost equal to 0.407 . The transient of the state-variables and the optimal input function are illustrated in Figs. 1, 2 respectively.

\section{Conclusions}

In this paper we have examined the convexity of the cost functional with respect to the input function of a particular class of positive systems with Markovian jumps. Such systems are characterized by the fact that the control variables affect the diagonal entries only, and these entries are convex functions of the control variables. The convexity property of the cost is important since is sufficient to guarantee the optimality of solutions satisfying the 


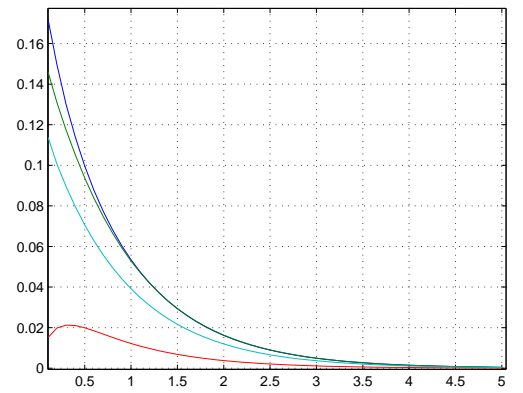

Figure 1. State variables of the nonlinear system (optimal mixed control for the linearized system)

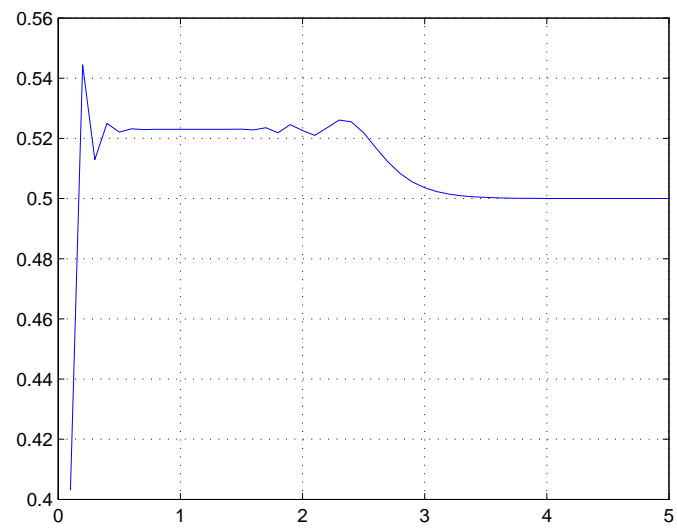

Figure 2. Optimal control variable associated with the worst initial state

Pontragyn minimum principle in a optimal control context. This fact opens the way to the use efficient computational methods to find the optimal control. On the other hand, the class of systems considered is amenable to characterize important applications in the field of system biology. In particular, a simple example taken from a infective/susceptive epidemiological model is considered. Extensions of this work can consider questions such as: the search for different application models and the extension of the theory to a wider class of positive nonlinear dynamic systems.

\section{REFERENCES}

[1] P. Bolzern, P. Colaneri, and G. De Nicolao, "Stochastic stability of positive Markov jump linear systems", Automatica, Vol. 50, 4, pp. 1181-1187, 2014. 
[2] F. Blanchini, P. Colaneri, R. Middleton, "A convexity result for the optimal control of a class of positive nonlinear systems", Proceedings of the 19th IFAC World Congress, pp. 1507-1512, 2014.

[3] P. Bolzern, P. Colaneri, "Positive Markov Jump Linear Systems", Foundations and Trends® in Systems and Control: Vol. 2: No. 3-4, pp 275-427. http://dx.doi.org/10.1561/2600000006.

[4] F. Blanchini, P. Colaneri, M.E. Valcher, "Switched linear positive systems", Foundations and Trends@ in Systems and Control, Vol. 2, N. 2, pp 101-273. http://dx.doi.org/10.1561/2600000005.

[5] F. Blanchini, P. Colaneri, M.E. Valcher, "Co-Positive Lyapunov Functions for the Stabilization of Positive Switched Systems", IEEE Transactions on Automatic Control, Vol. 57, N. 12, pp. 3038-3050, 2012.

[6] P. Bolzern, P. Colaneri, and G. De Nicolao, "Stabilization via switching of positive Markov jump linear systems", Proceedings of the 53nd IEEE Conference on Decision and Control, pages=-, 2014.

[7] A. Bressan, B. Piccoli, Introduction to Mathematical Control Theory, AIMS Series on Applied Mathematics, Philadelphia, 2007.

[8] C.G. Cassandras, D.L. Pepyne, and Y. Wardi, "Optimal control of a class of hybrid systems", IEEE Transactions on Automatic Control, Vol. 46, N. 3, pp. 398-415, 2001.

[9] P. Colaneri, R.H. Middleton, Z. Chen, D. Caporale, and F. Blanchini, "Convexity of the cost functional in an optimal control problem for a class of positive switched systems", Automatica, Vol. 50, pp. 1127-1243, 2014.

[10] P. Bolzern, P. Colaneri, G. De Nicolao, "On almost sure stability of continuous-time Markov Jump Linear Systems", Automatica, Vol. 42, pp. 983-988, 2006.

[11] C. G. Cassandras and S. Lafortune, Introduction to Discrete Event Systems, Springer, 2008.

[12] A.V. Dmitruk, and A.M. Kaganovich, "Maximum principle for optimal control problems with intermediate constraints", Computational Mathematics and Modeling, Vol. 22, N. 2, pp. 180-215, 2011.

[13] O.L. do Valle Costa, M.D. Fragoso, and M.G. Todorov, Continuous-Time Markov Jump Linear Systems, Springer, Heidelberg, New York, Dordrecht, London, 2012.

[14] L. Farina, and S. Rinaldi Positive linear systems: Theory and applications, Wiley New York, 2000.

[15] E. Hernandez-Vargas, P. Colaneri, R.H. Middleton, and F. Blanchini "Discrete-time control for switched positive systems with application to mitigating viral escape", International Journal of Robust and Nonlinear Control, Vol. 21, N. 10, pp. 1093-1111, 2011.

[16] E. Hernandez-Vargas, P. Colaneri, and R.H. Middleton, "Optimal therapy scheduling for a simplified HIV infection model", Automatica, Vol. 49, N. 9, pp. 2874-2880, 2013.

[17] K.J. Kushner, Stochastic Stability and Control, Springer New York, 1967.

[18] M. Margaliot, "Stability analysis of switched systems using variational principles: An introduction, Automatica, Vol. 42, N. 12, pp. 2059-2077, 2006.

[19] Y. Moreno, R. Pastor-Satorras, and A. Vespignani, "Epidemic outbreaks in complex heterogeneous networks", European Physical Journal B-Condensed Matter and Complex Systems, Vol. 26, N. 4, pp. 521-529, 2002.

[20] M.A. Rami, V.S Bokharaie, O. Mason, and F.R. Wirth, "Stability Criteria for SIS Epidemiological Models under Switching Policies", Discrete and continuous dynamical systems, Vol. 19, N. 9, pp. 2865-2887, 2014.

[21] L. Rapoport, "Asymptotic stability and periodic motions of selector-linear differential inclusions", Robust control via variable structure and Lyapunov techniques, pp. 269-285, 1996.

[22] P. Bolzern P. Colaneri, "Positive Markov Jump Linear Systems", Foundations and Trends in Systems and Control, 2015 . 
[23] P. Bolzern P. Colaneri, G. De Nicolao, "Stabilization via switching of positive Markov jump linear systems", 53rd IEEE Conference on Decision and Control, pp. 2359-2365, 2014.

[24] A. Doucet, N.J. Gordon, V. Krishnamurthy, "Particle filters for state estimation of jump Markov linear systems", IEEE Trans. on Signal Processing, Vol. 49, pp. 613-624, 2011.

[25] J.C. Geromel, G.S. Deaecto, P. Colaneri, "Minimax control of Markov jump linear systems ", Int. J. on Adaptive Control and Signal Processing, DOI: 10.1002/acs.2575, 2015.

[26] P. Colaneri and R. Middleton and Z. Chen and D. Caporale and F. Blanchini, "Convexity of the cost functional in an optimal control problem for a class of positive switched systems", Automatica, Vol. 50, pp. 1227-1234, 2014.

[27] E. A. Hernandez-Vargas, P. Colaneri, R. H. Middleton, "Switching Strategies to Mitigate HIV Mutation", IEEE Trans. Control Systems technology, Vol. 22, pp. 1623-1628, 2014. 